\title{
Desarrollo de competencias de liderazgo docente en alumnos del grado universitario de Educación
}

\author{
María Fernanda Gambarini Duarte, Soraya Muñoz Pérez ${ }^{b}$, María Crespí Ruperez \\ ${ }^{a}$ Universidad Francisco de Vitoria, Madrid, España, m.gambarini@ufv.es, ${ }^{b}$ Universidad Francisco de \\ Vitoria, Madrid, España, s.munoz@ufv.es, ${ }^{\mathrm{c}}$ Universidad Francisco de Vitoria, Madrid, España, \\ maria.crespi@ufv.es.
}

\begin{abstract}
Resumen
Nuestro objetivo es mostrar una experiencia de desarrollo de competencias de liderazgo en alumnos de los Grados en Educación primaria e infantil necesarios para ser un buen docente. Usando la metodología de trabajo cooperativo, se plantea a los alumnos la realización de un proyecto transversal donde se aúna el uso de las tecnologías, el acompañamiento individual y grupal por parte del equipo docente $y$ donde se integran conocimientos, actitudes y aptitudes de la asignatura de primero "Técnicas de Comunicación Eficaz”. El fin último es el análisis de las competencias docentes en búsqueda de la excelencia docente.
\end{abstract}

Palabras clave: Competencias, liderazgo, acompañamiento,comunicación

\section{Introdución}

En el marco Europeo de Estudios Universitarios, «....se apuesta por el concepto de aprender a aprender...se trata de potenciar el concepto de long life learning...» Para ello se establece un sistema marco de referencia de cualificaciones (EQF) centrado en los resultados de aprendizaje: lo que la persona sabe, comprende y es capaz de hacer. Se promueve por tanto un modelo centrado en el aprendizaje y en el alumno como protagonista; y en el que los resultados de aprendizaje están en clave de competencia .

En ese escenario competencial, desde la asignatura Técnicas de Comunicación Eficaz, impartida en los grados de educación infantil y primaria, se pretende dar respuesta a las demandas formativas en clave de competencia por parte de la sociedad del siglo XXI. Demandas por tanto, que hablan de profesionales de la educación competentes técnica y personalmente, con un "saber ser" docentes. Con un tipo de liderazgo que va más alla de "la capacidad de conducir personas, mediante una relación de influencia, hacia unos 
objetivos o metas". Como afirma Gil (2003), el docente es guía «...de un proceso particular de influencia social, guiado por un propósito moral con el fin de lograr objetivos educativos utilizando los recursos del aula.». Es por tanto un tipo de liderazgo definido por una relación de influencia personal, que basa su ascendiente en una forma de ser docente, y no simplemente de estar en el marco de la labor docente.

Ese liderazgo docente requiere de una serie de habilidades personales, ligadas fundamentalmente a la competencia de la comunicación eficaz como vía para el desarrollo óptimo de esa relación de influencia personal.

\section{Objetivos}

Desde la asignatura de Técnicas de Comunicación Eficaz, con un acompañamiento individual y grupal a los alumnos, se aspira a favorecer el desarrollo de habilidades asociadas a la competencia de comunicación eficaz. A través de una experiencia de aprendizaje basada en proyectos, se pretende promover en el alumno de los grados de Educación Infantil y Primaria un liderazgo intrapersonal que favorezca, el día de mañana, un liderazgo positivo en la relación con sus alumnos.

El tipo de liderazgo está íntimamente relacionado con la inteligencia intrapersonal que, según la aportación de Howard Gardner en su modelo de inteligencias múltiples, consiste en la inteligencia privada, es decir, contar con aquellas capacidades que permiten a la persona tener un modelo veraz y fiable de sí misma, que le dotan de la posibilidad de acceder a su mundo interior conociendo, aceptando y discriminando sus emociones a fin de orientar eficazmente su conducta.

Para desarrollar este liderazgo intrapersonal se propone a los alumnos de los grados de infantil y primaria la realización de un "Proyecto Transversal" que pretende potenciar el aprendizaje colaborativo, definido como el intercambio y cooperación social entre grupos de estudiantes para el propósito de facilitar la toma de decisiones y/o la solución de problemas. La colaboración entre aprendices les permite compartir hipótesis, enmendar sus pensamientos, y trabajar mediante sus discrepancias cognitivas. (Ralph y Yang, 1993)

Estos proyectos son el diseño de una propuesta de vía de comunicación eficaz familiaescuela, en el caso de infantil, y elaboración de un plan de formación a docentes en habilidades de comunicación eficaz, en el caso de primaria.

2.1- Objetivos generals para el Grado de Educación Infantil y Primaria

$\checkmark$ Aplicar las competencias intra e interpersonales propias de la profesión docente.

$\checkmark$ Demostrar el manejo de las técnicas y herramientas de comunicación eficaz en la relación con sus iguales. 


\section{2- Objetivos específicos}

Para el Grado de Educación Infantil y Primaria:

$\checkmark \quad$ Identificar las condiciones de una comunicación eficaz en el ámbito educativo.

$\checkmark$ Determinar las habilidades docentes en comunicación eficaz.

$\checkmark \quad$ Establecer el modelo de comunicación existente entre los agentes involucrados en el proceso de enseñanza-aprendizaje, en la sociedad del siglo XXI.

$\checkmark \quad$ Para el grado de Educación infantil: diseñar y planificar una vía de comunicación que favorezca la relación de confianza familia-escuela en su responsabilidad compartida y complementaria de educar, en el grado de infantil.

$\checkmark \quad$ Para el grado de Educación primaria: diseñar y planificar un plan de formación del profesorado en aquellas habilidades que les faciliten una comunicación eficaz.

\section{Metodología}

Se ha utilizado una metodología de Aprendizaje Basada en Proyectos implementada en los grados de educación infantil y primaria desde el año 2015 con una muestra de 100 alumnos. Para llevarla a cabo, se requiere un acompañamiento integral del alumno, que se concreta en dos ámbitos, tal como se detalla a continuación:

En ambos grupos manejamos una metodología común, auque en cada grado el Proyecto Trasversal gire en torno a un tema diferente. Así mismo, los resultados que esperamos en ambos grados son se refierien al proceso de adquisición y desarrollo de competencias de liderazgo docente, aunque el tema del Proyecto Trasversal no sea el mismo.

3.1- Acompañamiento integral al alumno: Entendemos el acompañamiento integral del alumno como el interés genuino y permanente por detectar el potencial del alumno y desarrollarlo al máximo, apoyándole en la obtención de los mejores resultados académicos, su formación integral, su integración en la sociedad y su felicidad.

3.1.1- Acompañamiento individual: con el objetivo de favorecer el desarrollo de habilidades de liderazgo intrapersonal (autoconocimiento, dominio personal e inteligencia emocional), cada alumno cuenta con la figura de un mentor que en su calidad de docente, acompaña al alumno durante 6 sesiones regladas en el proceso de adquisición y desarrollo de las mismas.

Este acompañamiento individual se vincula y articula con el acompañamiento, que en el aula y a través del Proyecto transversal, el profesor encargado de la asignatura realiza con el grupo de alumnos. 


\subsection{2- Acompañamiento grupal}

Con una metodología de trabajo cooperativo, donde la constitución de los equipos se realiza aleatoriamente, se genera el escenario adecuado para que los alumnos ejerciten las habilidades intrapersonales que han trabajado individualmente en compañia de su mentor.

Este contexto grupal permite al profesor de aula acompañar a cada uno de los equipos en su proceso de adquisión de habilidades de liderazgo interpersonales (escucha activa, empatía, comunicación asertivida, resolución de conflictos y trabajo en equipo).

\section{2- Entorno virtual b-learning}

Como sistema de apoyo se utiliza metodologia flippedclassroom y de gamificación. A través de la plataforma Wikispaces, se asigna una wiki a cada equipo y se van regulando las distintas fases del Proyecto. El producto que se recoge a partir de dichas fases son el resultado de investigación individual y grupal de los alumnos a partir de videos, artículos, miniquest, encuestas elaboradas y realizadas por los propios alumnos. Ambos proyectos se estructuran en las siguientes fases:

\section{Fase Exploratoria individual:}

Definición y justificación del marco teórico en el que se va sustentar la investigación (establecer y definir las habilidades de comunicación imprescindibles para una docencia eficaz).

\section{Fase de propuesta individual:}

Presentar y justificar con rigor académico la propuesta individual al equipo.

\section{Fase de ideación y desarrollo:}

Se acuerda una propuesta de equipo y se procede a la elaboración y desarrollo del Proyecto.

\section{Fase de exposición y defensa del Proyecto de equipo.}

Se presenta la memoria final del Proyecto y se defiende ante un tribunal constituido por el equipo docente de la asignatura.

La plataforma Moodle se utiliza para poner a disposición del alumno el material que contribuye a aumentar la base de conocimientos teóricos sobre las competencias. Así mismo, las tareas propuestas y la participación en los distinto foros promueve la reflexión crítica del alumno y el desarrollo de destrezas en la realización de trabajos escritos, en el análisis de la información y en el uso del entorno virtual. 


\section{Resultados}

Los resultados que se aportan recogen los datos desde el año 2015 hasta el 2018. Estos datos son fruto de una evaluación que recoge el grado en el que la metología anteriormente descrita, incide en el proceso de adquisición y desarrollo de competencias de liderazgo docente.

En la valoracion del nivel competencial se han tomado en consideración los tres dominios de aprendizaje que componen una competencia: conocimientos, habilidades y actitudes.

Se presentan tablas con la evolución de los resultados académicos de los alumnos a lo largo de estos cursos académicos (gráfico 1).

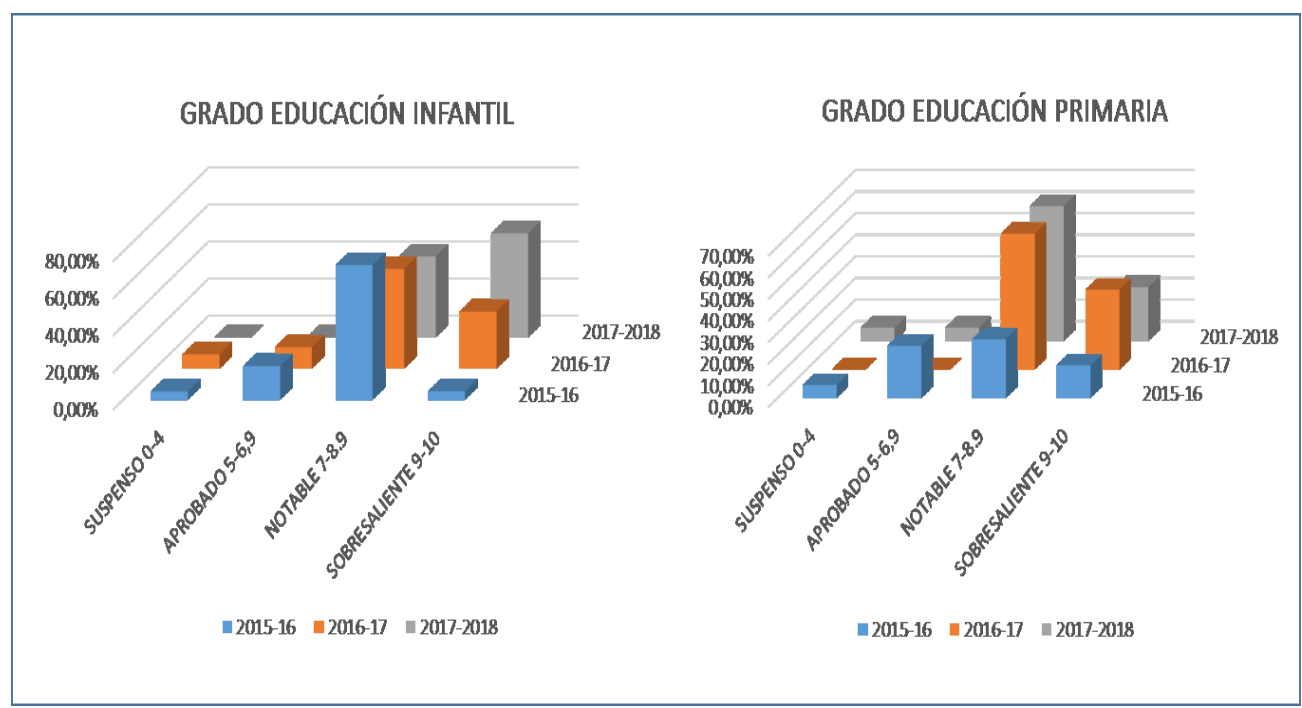

Grafico 1. Evolución notas grados infantil y primaria. Fuente: Actas de calificación de la asignatura Técnicas de Comunicación Eficaz. Universidad Francisco de Vitoria.

Otra fuente de información proviene de los resultados de CEDA, (Cuestionario de Evalaución Docente por el Alumno) que es el instrumento de medida utilizado en la universidad para medir la satisfacción de los alumnos con la labor de sus docentes. Así pues, se presentan los valores de autopercepcion del alumno sobre su desarrollo competencial según los items de resultados recogidos en el mencionado CEDA, representados en la siguiente tabla. 


\begin{tabular}{|l|c|c|c|}
\hline \multicolumn{1}{|c|}{ ITEMS } & $\mathbf{2 0 1 5 - 1 6}$ & $\mathbf{2 0 1 6 - 1 7}$ & $\mathbf{2 0 1 7 - 1 8}$ \\
\hline $\begin{array}{l}\text { He adquirido competencias y destrezas que me permiten resolver futuras } \\
\text { situaciones laborales. }\end{array}$ & 5,381 & 5,480 & 5,357 \\
\hline La formación recibida en la asignatura contribuye a mi desarrollo personal. & 5,619 & 5,520 & 5,571 \\
\hline La formación recibida contribuye a aumentar mi compromiso con los demás. & 5,476 & 5,440 & 5,500 \\
\hline
\end{tabular}

Tabla 1. Resultados ítems basados en el Cuestionario evaluación docente CEDA. Fuente: Cuestionario Evaluación docencia universitaria. Universidad Francisco de Vitoria.

Adicionalmente, durante el curso 2017/2018, se realizan dos encuestas, una al inicio del recorrido de la asignatura y otra al final del mismo. En ellas se pide a los alumnos del grado de infantil y de primaria que valoren su nivel de desarrollo competencial en base a los ámbitos recogidos en la tabla 2 y cuyos resultados se aportán en los gráficos 2 y 3:

\begin{tabular}{|c|l|}
\hline \multirow{2}{*}{ AUTOCONOCIMIENTO } & Item 1: Capacidad de reflexión sobre la propia acción \\
& Item 2: Actitud proactiva ante el propio error \\
& Item 3: Flexibilidad y apertura ante el feed-back del otro \\
& Item 4: Actitud receptiva ante la ayuuda del otro. \\
\hline \multirow{2}{*}{ RESOLUCIÓN DE CONFLICTOS } & Item 1: Actitud proactiva ante el conflicto \\
& $\begin{array}{l}\text { Item 2: Capacidad de análisis del conflicto } \\
\text { Item 3: Capacidad de reflexión sobre alternativas resolución }\end{array}$ \\
& Item 4: Capacidad de ponderación entre alternativas. \\
\hline \multirow{2}{*}{ DOMINIO PERSONAL } & Item 1: Capacidad de control estado emocional. \\
& Item 2: Capacidad de control del estres. \\
& Item 3: Capacidad de reacción ante el estado emocional. \\
& Item 4: Capacidad de control emocional ante el conflicto \\
\hline \multirow{2}{*}{ EMPATÍA } & Item 1: Capacidad de comprender la situación del otro. \\
& Item 2: Capacidad para tener en cuenta sentimientos del otro. \\
& Item 3: Capacidad de comunicación empática. \\
& Item 4: Capacidad de reflexión sobre impacto propia acción en el otro. \\
\hline \multirow{2}{*}{ ESCUCHA } & Item 1: Actitud apertura y flexibilidad ante la opinion del otro. \\
& Item 2: Voluntad de comunicación eficaz . \\
& Item 3: Capacidad de adaptar el estilo de comunicación. \\
& Item 4: Capacidad de comunicación clara, sincera y efectiva. \\
\hline & Item 1: Capacidad de escuchar sin interrumpir . \\
& Item 2: Capacidad para attender al lenguaje no verbal del otro. \\
& Item 3: Capacidad para resumir el mensaje del emisor . \\
& Item 4: Capacidad para solicitar ampliacion de información del emisor. \\
\hline
\end{tabular}

Tabla 2. Cuestionario autoevaluación desarrollo competencial. Fuente: elaboración propia. 


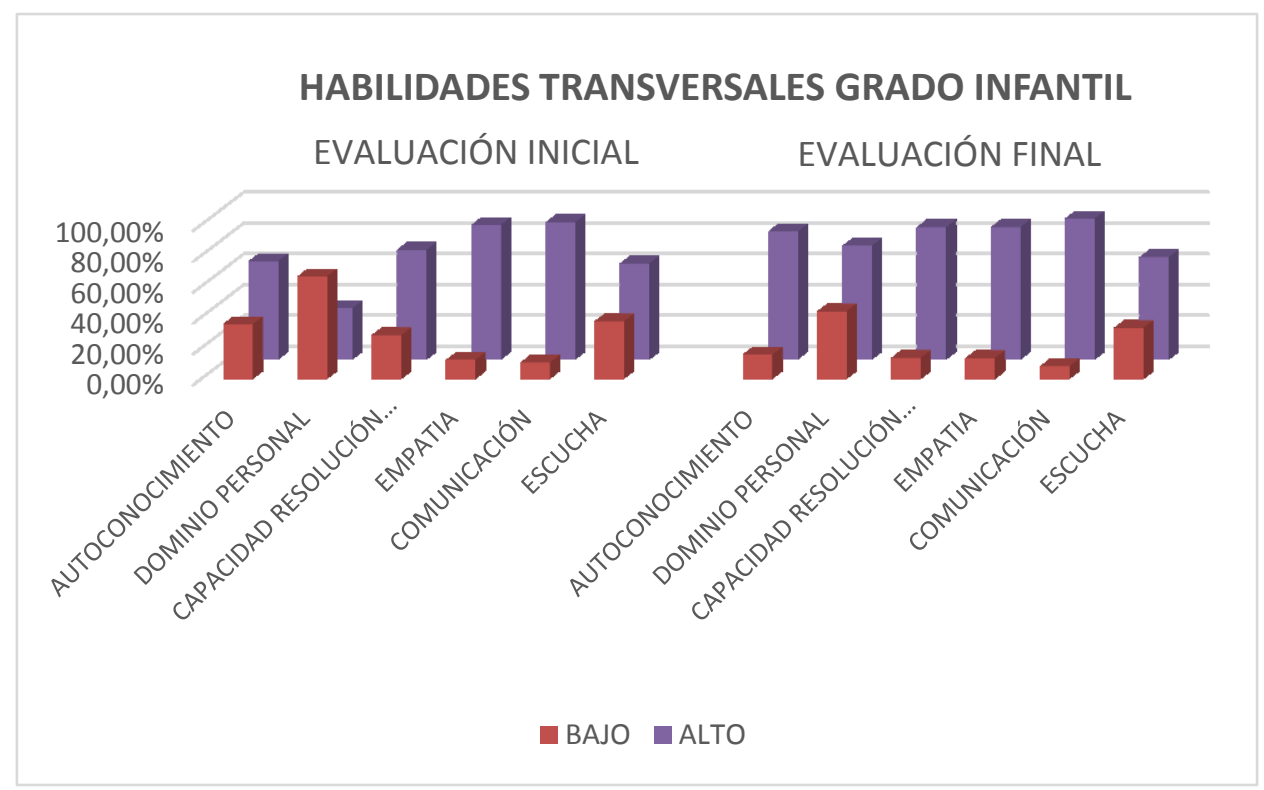

Gráfico 2. Grado infantil. Fuente: elaboración propia.

\section{HABILIDADES TRANSVERSALES GRADO PRIMARIA \\ EVALUACIÓN INICIAL EVALUACIÓN FINAL}

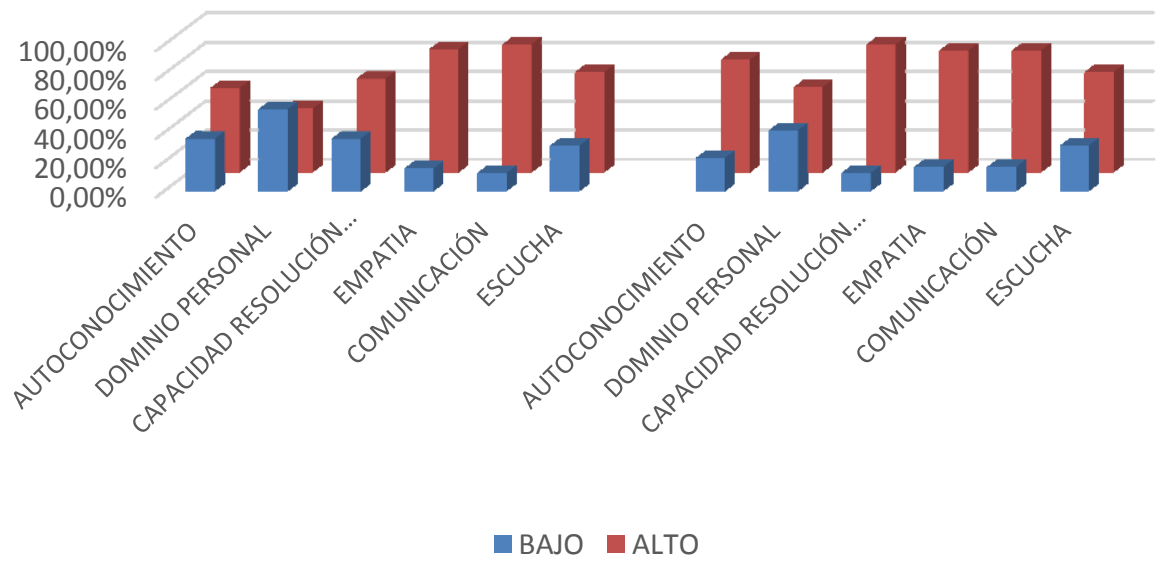

Gráfico 3. Grado primaria. Fuente: elaboración propia. 


\section{Discusión}

A partir de los resultados presentados se puede apreciar:

1. Los resultados académicos obtenidos en la asignatura arrojan un incremento de las calificaciones de notable y sobresaliente en los periodos académicos 2016-2017 y 2017-2018. Este incremento se aprecia de forma más palpable en el grado de primaria, .Gráfico 1.

2. Los items del CEDA que recogen la percepción de los resultados en el cuestionario de evaluación docente CEDA ha sido expresada como excelente en el cuestionario de evaluación CEDA, al obtener evaluaciones superiores a 5 sobre 6 (escala Likert). Tabla 1.

3. En el grado de infantil, la comparativa entre los resultados obtenidos en las encuestas de autoevaluación inicial y final reflejan un incremento en el autoconocimiento del $19 \%$ y de dominio personal de un $40 \%$. El incremento en capacidad de resolución de conflictos es de un $2.65 \% \mathrm{y}$ en el de habilidades de comunicación (comunicación, empatía y escucha) es de un $2 \%$. Gráfico 2 .

4. En el grado de primaria, la comparativa entre los resultados obtenidos en las encuestas de autoevaluación inicial y final reflejan un incremento en el autoconocimiento del $19.27 \%$ y de dominio personal de un $14.55 \%$. El incremento en capacidad de resolución de conflictos es de un $23.44 \%$. En el ámbito de las habilidades de comunicación (comunicación, empatía y escucha), en el caso del grado de primaria, se refleja una disminución de un $1.73 \%$. Gráfico 3.

\section{Conclusión}

A partir de los resultados presentados se puede apreciar:

Que el acompañamiento individual y grupal al alumno de los grados de infantil y primaria ha favorecido la mejora en los resultados académicos obtenidos en la asignatura; principalmente en el grado de primaria en los periodos académicos 2016-2017 y 2017-2018 .Gráfico 1.

El nivel de satisfacción de los alumnos con respecto al impacto de la metodología en el nivel de desarrollo competencial ha sido expresada como excelente en el cuestionario de evaluación CEDA, al obtener evaluaciones superiores a 5 sobre 6 (escala Likert). Los items seleccionados se han considerado por ser los que refieren la percepción del alumno sobre la incidencia de la metodologíoa utilizada en su proceso personal de desarrollo en Habilidades de liderazgo docente. Tabla 1. 
La comparativa entre los resultados obtenidos en las encuestas de autoevaluación inicial y final reflejan que el alumno percibe una mejora en habilidades de liderazgo intrapersonal (autoconocimiento, dominio personal) e interpersonal (capacidad de resolución de conflictos, comunicación y empatía). Gráficos 2 y 3. La disminución en el porcentaje que se aprecia en la autoevaluación final de los alumnos del grado de primaria sobre las habilidades de comunicación se atribuye, tras conversación con los alumnos, a lo siguiente: el mayor grado de conocimiento personal les hace caer en la cuenta de sus puntos de mejora en cuanto a la comunicación con el otro.

Por otro lado, el hecho de que estos resultados en la autoevaluación final coincidan con las puntuaciones del cuestionario CEDA de evaluación docente, hacen pensar en la relación directa entre la metodología utilizada en la asignatura de Técnicas de Comunicación Eficaz y la autopercepción por parte del alumno de una mejora en sus habilidades personales. Los resultados académicos obtenidos por los alumnos aportan evidencia y objetividad a dicha percepción.

\section{Referencias}

Álvarez De Mon, S. (2004) Carácter del liderazgo: Lecciones desde la adversidad. Revista Empresa y Humanismo Vol. V, no 1/02, , pp. 23-46 Fecha de consulta: 18 de Junio de 2018 (https://goo.gl/P3xn3Q)

Bañares, L El carácter sistémico del liderazgo. Anuario Filosófico 1996, n 26, p 386 Servicio Publicaciones Universidad de Navarra. Pamplona. Fecha de consulta: 20 de Junio de 2018 (https://goo.gl/oChJZC)

Cardona, P., Rey, C., El liderazgo centrado en la misión: Cómo lograr el liderazgo en toda la organización, IESE, OP-08-4, 01/2008, p. 2. Fecha de consulta: 18 de Junio de 2018 (https://goo.gl/e2mRnG)

Cerrillo, M.R. (2003), Educar en valores, misión del profesor" Tendencias pedagógicas 8, pp. 59-68. Fecha de consulta: 20 de Junio de 2018 (https://goo.gl/dsGbdi)

Delgado Fernández, M., \& Solano González, A. (2009). Estrategias didácticas creativas en entornos virtuales para el aprendizaje. Revista Electrónica "Actualidades Investigativas en Educación", 9 (2), 1-21. Fecha de consulta: 29 de Junio de 2018 ( https://goo.gl/v41F7J)

Gardner, H. (2016). Estructuras de la mente: la teoría de las inteligencias múltiples. Fondo de cultura económica.

Gargallo, B., Suárez-Rodríguez, J., Garfella, P.R., Fernández-March, A. (2011). El cuestionario CEMEDEPU. Un instrumento para la evaluación de la metodología docente y evaluativa de los 
profesores universitarios. ESE. Estudios sobre educación. 21, 49-7. Fecha de consulta: 29 de Junio de 2018 (https://goo.gl/ESmWES)

Gil, F. y otros. (2013) El liderazgo educativo en el contexto del aula. En J. Argos y P. Ezquerra (Eds.) Liderazgo y educación. Universidad de Cantabria, ISBN: 978-84-86116-80-4 pp. 99-124.

Estilos de Enseñanza (ESEE). Enseñanza \& Teaching, 31, 1-2013, pp 181-198. Fecha de consulta: 18 de Junio de 2018 (https://goo.gl/gxNqro)

Guardian-Fernandez, A. (2007): El Paradigma Cualitativo en la investigación socio-educativa. Investigación y Desarrollo. Educativo Regional (IDER) Fecha de consulta: 4 de Junio de 2018 (https://goo.gl/sW6exp)

Mañú, J. M., Goyarrola, I. (2011) Docentes competentes: por una educación de calidad. Madrid. Narcea Ediciones.

Ralph, Edwin G. y Yang, Baiyin. (1993). Beginning teachers' utilization of instructional media: A Canadian case study, ETTI Educational \& training technology International, 30 (4), 299-318. Fecha de consulta: 17 de Junio de 2018 (https://bit.ly/2KqngXV)

Ramos, J. M. G. (1997). Valoración de la competencia docente del profesor universitario. Una aproximación empírica. Revista complutense de educación, 8, 81 Fecha de consulta: 4 de Junio de 2018 (https://goo.gl/BPZaR1)

Salovey, P. y Mayer, J.D. (1990). Emotional intelligence. Imagination, Cognition, and Personality, 9, pp 185-211. Fecha de consulta: 20 de Junio de 2018 (https://doi.org/10.2190/DUGG-P24E$\underline{52 \mathrm{WK}-6 \mathrm{CDG})}$

Sonnenfeld, A. (2010) Liderazgo ético. La sabiduría de decidir bien. Madrid. Ediciones Encuentro, S. A. y Nueva Revista. Madrid 